\title{
A new basis set for the description of electrons in superconductors
}

\author{
J. E. Hirsch \\ Department of Physics, University of California, San Diego \\ La Jolla, CA 92093-0319
}

\begin{abstract}
In the usual description of electrons in metals and superconductors, the single electron states are assumed to satisfy Bloch's theorem. This is because the electron-ion interaction is privileged over the electron-electron interaction. However the theory of hole superconductivity proposes that in the transition to superconductivity carriers 'undress' from the electron-ion interaction. I propose here a new basis set to describe electrons in the superconducting state that does not satisfy Bloch's theorem but instead is designed to optimize the electron-electron interaction. The new basis set favors a superconducting state where a spin current exists and where states near the bottom of the band become partially occupied, as predicted by the theory of hole superconductivity.
\end{abstract}

PACS numbers:

\section{INTRODUCTION}

It is generally assumed that electron states in a periodic solid obey Bloch's theorem[1]. That is, a wavevector $\mathbf{k}$ characterizes the single electron orbital $\psi_{n \mathbf{k}}(\mathbf{r})$ which obeys the relation

$$
\psi_{n \mathbf{k}}(\mathbf{r}+\mathbf{R})=e^{i \mathbf{k} \cdot \mathbf{R}} \psi_{n \mathbf{k}}(\mathbf{r})
$$

where the index $n$ (band index) differentiates different states with the same Bloch wavevector $\mathbf{k}$. In Eq. (1), R is any lattice translation vector that leaves the electronion potential invariant:

$$
U_{e-i}(\mathbf{r}+\mathbf{R})=U_{e-i}(\mathbf{r})
$$

Electron-electron interactions are assumed to convert the single-particle electron-ion potential to a new 'effective' potential $U_{\text {eff }}$ that includes electron-electron interaction direct, exchange and correlation effects (e.g. the KohnSham potential in density-functional theory) [2]. The effective potential still obeys

$$
U_{e f f}(\mathbf{r}+\mathbf{R})=U_{e f f}(\mathbf{r})
$$

with the same lattice vectors $\mathbf{R}$, and the corresponding single particle orbitals (e.g. Kohn-Sham orbitals) still obey Bloch's theorem Eq. (1).

Similarly, in the generally accepted conventional theory of superconductivity (BCS theory) [3] the many-body wavefunction is written as

$$
\left|\Psi>_{B C S}=\prod_{\mathbf{k}}\left(u_{\mathbf{k}}+v_{\mathbf{k}} c_{\mathbf{k} \uparrow}^{\dagger} c_{-\mathbf{k} \downarrow}^{\dagger}\right)\right| 0>
$$

where the index $\mathbf{k}$ is the Bloch wavevector of the state where the single electron of spin $\sigma$ is created by the operator $c_{\mathbf{k} \sigma}^{\dagger}$. The normal state of the system can be expressed in the form Eq. (4) with $u_{\mathbf{k}} v_{\mathbf{k}}=0$ and $v_{\mathbf{k}}=0$ or $u_{\mathbf{k}}=0$ depending on whether $\epsilon_{\mathbf{k}}<\epsilon_{F}$ or $\epsilon_{\mathbf{k}}>\epsilon_{F}$ with $\epsilon_{F}$ the Fermi energy. The superconducting state instead is characterized by $u_{\mathbf{k}} v_{\mathbf{k}} \neq 0$ for some values of $\mathbf{k}$. For the case of nearly free electron metals, the Bloch states are often simply taken to be free electron plane wave states.

However, it is not necessarily true that electron states in a periodic electron-ion potential have to obey Bloch's theorem. Consider a case where the electron-electron interaction completely overwhelms the electron-ion interaction. The electronic spatial arrangement that minimizes the energy may be an electronic lattice (Wigner crystal, incommensurate charge density wave) with a lattice structure that has no relation with the underlying ionic structure. For example, the electronic structure may be hexagonal and the underlying ionic lattice cubic. In such situation the single particle states describing the electrons will not obey Bloch's theorem Eq. (1).

We have recently argued that experimental evidence clearly indicates that in the transition to superconductivity the carriers undergo a wavelength expansion and end up completely 'undressed' from the electron-ion interaction, so that they no longer 'see' the discrete nature of the electron-ion potential [4, 5]. It is natural to conclude that the electron states describing the superfluid will no longer obey Bloch's theorem.

Furthermore, we have recently proposed that the superconducting state should be understood as a (distorted) 'mirror image' of the normal state, where the 'mirror' switches the role of the electron-ion and electronelectron interactions [6]. We argued that in the superconducting state, the electron-electron interaction is privileged over the electron-ion interaction and the manybody wavefunction optimizes the electron-electron interaction, with the electron-ion interaction being nonoptimized. It is natural that such a state should be described by a single electron basis that does not satisfy Bloch's theorem. But what is the optimal basis to describe such state?

The new single-particle basis states that we propose here are defined by the relation

$$
\tilde{\varphi}_{\mathbf{k}}(\mathbf{r})=\frac{\varphi_{\mathbf{k}}(\mathbf{r})+i \varphi_{-\mathbf{k}}(\mathbf{r})}{\sqrt{2}}
$$

where $\varphi_{\mathbf{k}}$ are the usual Bloch states for a given band. Note that $\left\{\tilde{\varphi}_{\mathbf{k}}\right\}$ is a complete orthonormal set if and only 
if $\left\{\varphi_{\mathbf{k}}\right\}$ is. The inverse relation is

$$
\varphi_{\mathbf{k}}(\mathbf{r})=\frac{\tilde{\varphi}_{\mathbf{k}}(\mathbf{r})-i \tilde{\varphi}_{-\mathbf{k}}(\mathbf{r})}{\sqrt{2}}
$$

Similarly we define the electron creation operators in the new states $\tilde{c}_{\mathbf{k} \sigma}^{\dagger}$ in terms of the Bloch creation operators $c_{\mathbf{k} \sigma}^{\dagger}$ by

$$
\begin{gathered}
\tilde{c}_{\mathbf{k} \sigma}^{\dagger}=\frac{c_{\mathbf{k} \sigma}^{\dagger}+i c_{-\mathbf{k} \sigma}^{\dagger}}{\sqrt{2}} \\
c_{\mathbf{k} \sigma}^{\dagger}=\frac{\tilde{c}_{\mathbf{k} \sigma}^{\dagger}-i \tilde{c}_{-\mathbf{k} \sigma}^{\dagger}}{\sqrt{2}}
\end{gathered}
$$

It is clear that our new basis functions do not satisfy Bloch's theorem, since

$$
\begin{aligned}
\tilde{\varphi}_{\mathbf{k}}(\mathbf{r}+\mathbf{R}) & =\frac{e^{i \mathbf{k} \cdot \mathbf{R}} \varphi_{\mathbf{k}}(\mathbf{r})+i e^{-i \mathbf{k} \cdot \mathbf{R}} \varphi_{-\mathbf{k}}(\mathbf{r})}{\sqrt{2}} \\
& \neq e^{i \mathbf{k} \cdot \mathbf{R}} \tilde{\varphi}_{\mathbf{k}}(\mathbf{r})
\end{aligned}
$$

Nevertheless, I will argue that the new basis has very interesting properties and is relevant to the description of the superconducting state.

\section{NATURE OF THE WAVE FUNCTIONS}

Consider the case where the Bloch functions are well approximated by plane wave states

$$
\varphi_{\mathbf{k}}(\mathbf{r})=\frac{1}{\sqrt{\Omega}} e^{i \mathbf{k} \cdot \mathbf{r}}
$$

Then

$$
\begin{gathered}
\tilde{\varphi}_{\mathbf{k}}(\mathbf{r})=\frac{1+i}{\sqrt{\Omega}} \cos \left(\mathbf{k} \cdot \mathbf{r}-\frac{\pi}{4}\right) \\
\tilde{\varphi}_{-\mathbf{k}}(\mathbf{r})=\frac{1+i}{\sqrt{\Omega}} \cos \left(\mathbf{k} \cdot \mathbf{r}+\frac{\pi}{4}\right) .
\end{gathered}
$$

describing standing waves. The values of $\mathbf{r}$ for which $\left|\tilde{\varphi}_{\mathbf{k}}(\mathbf{r})\right|^{2}$ is maximum satisfy

$$
\mathbf{k} \cdot \mathbf{r}-\frac{\pi}{4}=n \pi
$$

with $n$ integer, and for those $\mathbf{r}$ values

$$
\mathbf{k} \cdot \mathbf{r}+\frac{\pi}{4}=\left(n+\frac{1}{2}\right) \pi
$$

so that $\left|\tilde{\varphi}_{-\mathbf{k}}(\mathbf{r})\right|^{2}=0$. Consequently this will cause a strong reduction of the direct Coulomb repulsion between an electron in state $\tilde{\varphi}_{\mathbf{k}}$ and another electron in state $\tilde{\varphi}_{-\mathbf{k}}$ compared to the plane wave case where the density is uniform, as illustrated in Fig. 1. Conversely, the in-phase
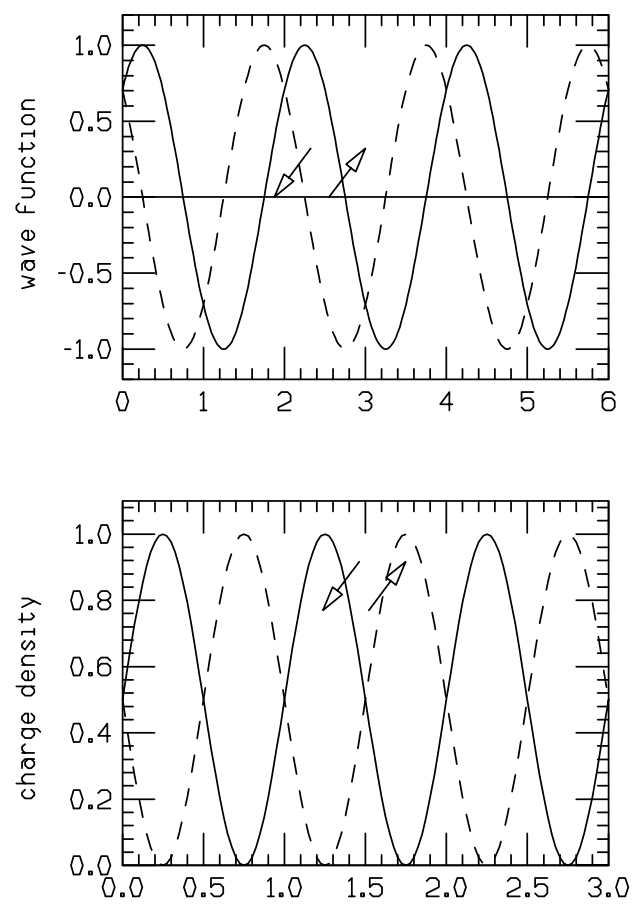

FIG. 1: Wave function amplitudes and charge densities for the standing waves associated with the operators $\tilde{c}_{\mathbf{k} \sigma}^{\dagger}$ (full lines) and $\tilde{c}_{-\mathbf{k},-\sigma}^{\dagger}$ (dashed lines), assuming the Bloch states are plane waves. Note that the charge densities avoid each other reducing the Coulomb repulsion.

modulation of the wavefunction amplitude for two electrons in the same state $\tilde{\varphi}_{\mathbf{k}}$ will give a strong enhancement of the direct Coulomb repulsion in that case compared to the plane wave case.

Similarly, consider a band described by the tight binding approximation with a single orbital $\phi_{i}(\mathbf{r})$ per site. The Bloch functions are

$$
\varphi_{\mathbf{k}}(\mathbf{r})=\frac{1}{\sqrt{N}} \sum_{i} e^{i \mathbf{k} \cdot \mathbf{R}_{i}} \phi_{i}(\mathbf{r})
$$

and the new orbitals are given by

$$
\begin{gathered}
\tilde{\varphi}_{\mathbf{k}}(\mathbf{r})=\frac{1+i}{\sqrt{N}} \sum_{i} \cos \left(\mathbf{k} \cdot \mathbf{R}_{i}-\frac{\pi}{4}\right) \phi_{i}(\mathbf{r}) \\
\tilde{\varphi}_{-\mathbf{k}}(\mathbf{r})=\frac{1+i}{\sqrt{N}} \sum_{i} \cos \left(\mathbf{k} \cdot \mathbf{R}_{i}+\frac{\pi}{4}\right) \phi_{i}(\mathbf{r})
\end{gathered}
$$

It is clear that for small $\mathbf{k}$ the situation is the same as for the plane-wave case: the values of $\mathbf{R}_{i}$ for which the amplitude is maximum in Eq. (12a) coincide (or nearly coincide) with those where the amplitude in Eq. (12b) is zero. However, this changes when the wavevector $\mathbf{k}$ becomes an appreciable fraction of a reciprocal lattice vector $\mathbf{G}$. We have

$$
\cos \left(\mathbf{k} \cdot \mathbf{R}_{i} \mp \frac{\pi}{4}\right)=\frac{1}{\sqrt{2}}\left(\cos \left(\mathbf{k} \cdot \mathbf{R}_{i}\right) \pm \sin \left(\mathbf{k} \cdot \mathbf{R}_{i}\right)\right)
$$


so that the amplitudes in Eq. (12) become the same when $\mathbf{k} \rightarrow \mathbf{G} / 2$, since the second term in Eq. (13) vanishes. Thus, we expect the direct Coulomb repulsion between an electron in state $\tilde{\varphi}_{\mathbf{k}}$ and one in state $\tilde{\varphi}_{-\mathbf{k}}$ to be reduced, and that between two electrons in the same state $\tilde{\varphi}_{\mathbf{k}}$ to be enhanced, when $\mathbf{k}$ is near the bottom of the band but not when $\mathbf{k}$ is near the top of the band.

\section{COULOMB REPULSION IN THE NEW BASIS}

The bare Coulomb interaction between opposite spin electrons in a plane wave basis is given by

$$
\begin{gathered}
V_{c}=\frac{1}{\Omega} \sum_{\mathbf{k}^{\prime} \mathbf{q}} V(\mathbf{q}) c_{\mathbf{k}+\mathbf{q} \uparrow}^{\dagger} c_{\mathbf{k}^{\prime}-\mathbf{q} \downarrow}^{\dagger} c_{\mathbf{k}^{\prime} \downarrow} c_{\mathbf{k} \uparrow} \\
V(\mathbf{q})=\frac{4 \pi e^{2}}{q^{2}}
\end{gathered}
$$

with $\Omega$ the volume of the system. The Coulomb interaction between an electron of spin $\uparrow$ in the plane wave state $\varphi_{\mathbf{k}}$ and an electron of spin $\downarrow$ in $\varphi_{\mathbf{k}}^{\prime}$

$$
\left|\mathbf{k} \uparrow, \mathbf{k}^{\prime} \downarrow>\equiv c_{\mathbf{k} \uparrow}^{\dagger} c_{\mathbf{k}^{\prime} \downarrow}^{\dagger}\right| 0>
$$

is simply

$$
\begin{aligned}
& <\mathbf{k} \uparrow, \mathbf{k}^{\prime} \downarrow\left|V_{c}\right| \mathbf{k} \uparrow, \mathbf{k}^{\prime} \downarrow>= \\
& \int d^{3} r d^{3} r^{\prime}\left|\varphi_{\mathbf{k}}(\mathbf{r})\right|^{2} \frac{e^{2}}{\left|\mathbf{r}-\mathbf{r}^{\prime}\right|}\left|\varphi_{\mathbf{k}^{\prime}}(\mathbf{r})\right|^{2}=\frac{1}{\Omega} V(0)(15
\end{aligned}
$$

independent of $\mathbf{k}$ and $\mathbf{k}^{\prime}$. The Coulomb interaction between an electron of spin $\uparrow$ in state $\tilde{\varphi}_{\mathbf{k}}$ and an electron of spin $\downarrow$ in state $\tilde{\varphi}_{\mathbf{k}}^{\prime}$ :

$$
\left|\tilde{\mathbf{k}} \uparrow, \tilde{\mathbf{k}}^{\prime} \downarrow>\equiv \tilde{c}_{\mathbf{k} \uparrow}^{\dagger} \tilde{c}_{\mathbf{k}^{\prime} \downarrow}^{\dagger}\right| 0>
$$

is given by

$$
\begin{aligned}
& <\tilde{\mathbf{k}} \uparrow, \tilde{\mathbf{k}}^{\prime} \downarrow\left|V_{c}\right| \tilde{\mathbf{k}} \uparrow, \tilde{\mathbf{k}}^{\prime} \downarrow>=\frac{1}{\Omega} V(0) \\
& +\frac{1}{2 \Omega} V(2 k)\left(-\delta_{\mathbf{k}^{\prime},-\mathbf{k}}+\delta_{\mathbf{k}^{\prime}, \mathbf{k}}\right)
\end{aligned}
$$

showing indeed a reduction for $(\mathbf{k} \uparrow,-\mathbf{k} \downarrow)$ in the new basis as well as an increased repulsion for $(\mathbf{k} \uparrow, \mathbf{k} \downarrow)$, since the Coulomb matrix element $V(2 k)$ is always positive. Note also that the effect is largest for small $k$.

Similarly consider the Coulomb interactions in the tight binding model. We will assume for simplicity that the only non-zero matrix element of the Coulomb interaction in the tight binding model is the onsite Coulomb repulsion $\mathrm{U}$ :

$$
U=\int d^{3} r d^{3} r^{\prime}\left|\varphi_{i}(\mathbf{r})\right|^{2} \frac{e^{2}}{\left|\mathbf{r}-\mathbf{r}^{\prime}\right|}\left|\varphi_{i}\left(\mathbf{r}^{\prime}\right)\right|^{2}
$$

The Coulomb repulsion between an electron in Bloch state $\mathbf{k}$ and one in Bloch state $\mathbf{k}^{\prime}$ is

$$
<\mathbf{k} \uparrow, \mathbf{k}^{\prime} \downarrow\left|V_{c}\right| \mathbf{k} \uparrow, \mathbf{k}^{\prime} \downarrow>=\frac{U}{N}
$$

independent of $\mathbf{k}$ and $\mathbf{k}^{\prime}$. Instead, in the new basis

$$
\begin{aligned}
& <\tilde{\mathbf{k}} \uparrow, \tilde{\mathbf{k}}^{\prime} \downarrow\left|V_{c}\right| \tilde{\mathbf{k}} \uparrow, \tilde{\mathbf{k}}^{\prime} \downarrow>= \\
& \quad \frac{U}{N^{2}} \sum_{i}\left(1+\sin \left(2 \mathbf{k} \cdot \mathbf{R}_{\mathbf{i}}\right)\right)\left(1+\sin \left(2 \mathbf{k}^{\prime} \cdot \mathbf{R}_{\mathbf{i}}\right)\right)
\end{aligned}
$$

showing in particular that the interaction is most reduced for $\mathbf{k}^{\prime}=-\mathbf{k}$, and most enhanced for $\mathbf{k}^{\prime}=\mathbf{k}$. However, for $\mathbf{k}$ and $\mathbf{k}^{\prime}$ denoting states near the top of the band, $\sim \mathbf{G} / 2$, with $\mathbf{G}$ a reciprocal lattice vector, the effect disappears, in agreement with the discussion in the previous section.

However, Eq. (19) appears to suggest that the Coulomb interactions are the same also for states around the half-filled band, e.g. $\mathbf{k}=\mathbf{G} / 4$. This is an artifact caused by keeping only the on-site Coulomb matrix element $U$. When considering other Coulomb matrix elements also it is found that the Coulomb interaction between states in the new basis and in the conventional basis become the same only when the states approach the top of the band and the wavevectors are connected by reciprocal lattice vectors.

Next we consider the Coulomb interaction in the triplet and singlet $(\mathbf{k} \uparrow,-\mathbf{k} \downarrow)$ pairs. We have

$$
\tilde{c}_{\mathbf{k} \uparrow}^{\dagger} \tilde{c}_{-\mathbf{k} \downarrow}^{\dagger}-\tilde{c}_{-\mathbf{k} \uparrow}^{\dagger} \tilde{c}_{\mathbf{k} \downarrow}^{\dagger}=c_{\mathbf{k} \uparrow}^{\dagger} c_{-\mathbf{k} \downarrow}^{\dagger}-c_{-\mathbf{k} \uparrow}^{\dagger} c_{\mathbf{k} \downarrow}^{\dagger}
$$

so that the triplet state in the new basis,

$$
\left|\tilde{t}>=\frac{\tilde{c}_{\mathbf{k} \uparrow}^{\dagger} \tilde{c}_{-\mathbf{k} \downarrow}^{\dagger}-\tilde{c}_{-\mathbf{k} \uparrow}^{\dagger} \tilde{c}_{\mathbf{k} \downarrow}^{\dagger}}{2}\right| 0>
$$

is identical to the triplet state in the conventional basis, $\mid t>$. The Coulomb interaction is

$$
<\tilde{t}\left|V_{c}\right| \tilde{t}>=<t\left|V_{c}\right| t>=\frac{1}{\Omega}(V(0)-V(2 k)) .
$$

The singlet states are different however, since

$$
\tilde{c}_{\mathbf{k} \uparrow}^{\dagger} \tilde{c}_{-\mathbf{k} \downarrow}^{\dagger}+\tilde{c}_{-\mathbf{k} \uparrow}^{\dagger} \tilde{c}_{\mathbf{k} \downarrow}^{\dagger}=i\left(c_{\mathbf{k} \uparrow}^{\dagger} c_{\mathbf{k} \downarrow}^{\dagger}+c_{-\mathbf{k} \uparrow}^{\dagger} c_{-\mathbf{k} \downarrow}^{\dagger}\right)
$$

and we have

$$
\begin{gathered}
<\tilde{s}\left|V_{c}\right| \tilde{s}>=\frac{V(0)}{\Omega} \\
<s\left|V_{c}\right| s>=\frac{1}{\Omega}(V(0)+V(2 k))
\end{gathered}
$$

so the Coulomb repulsion is smaller in the singlet state of the new basis than in the singlet state of the conventional basis. The average of Eqs. (22) and (24a) or (24b) yields Eq. (16b) or Eq. (15b) for $\mathbf{k}^{\prime}=-\mathbf{k}$, since the state $\mid \mathbf{k} \uparrow,-\mathbf{k} \downarrow>$ is a linear combination of singlet and triplet states with equal weight. 
A similar result holds in the tight binding model, where we have (assuming only on-site interactions)

$$
\begin{gathered}
<\tilde{t}\left|V_{c}\right| \tilde{t}>=<t\left|V_{c}\right| t>=0 \\
<\tilde{s}\left|V_{c}\right| \tilde{s}>=\frac{2 U}{N^{2}} \sum_{i} \cos ^{2} 2 \mathbf{k} \cdot \mathbf{R}_{i} \\
<s\left|V_{c}\right| s>=\frac{2 U}{N}
\end{gathered}
$$

Again the average of Eq. (25a) and Eqs. (25b) or (25c) yields Eq. (19) or (18) for $\mathbf{k}^{\prime}=-\mathbf{k}$.

\section{RELEVANCE TO SUPERCONDUCTIVITY}

Our findings in the previous sections suggest that the new basis is relevant to superconductivity. The fact that the BCS wavefunction Eq. (4) singles out pairs $(\mathbf{k} \uparrow,-\mathbf{k} \downarrow$ ) could naturally be explained by the fact that the direct Coulomb repulsion between an $\uparrow$-spin electron in state $\tilde{\varphi}_{\mathbf{k}}$ and a $\downarrow$-spin electron in $\tilde{\varphi}_{-\mathbf{k}}$ is particularly small.

Consider then as an alternative to the BCS wavefunction a wavefunction of the form Eq. (4) but with the new states instead of the Bloch states:

$$
\left|\Psi>_{n e w}=\prod_{\mathbf{k}}\left(u_{\mathbf{k}}+v_{\mathbf{k}} \tilde{c}_{\mathbf{k} \uparrow}^{\dagger} \tilde{c}_{-\mathbf{k} \downarrow}^{\dagger}\right)\right| 0>
$$

Deep in the Fermi sea, $u_{\mathbf{k}}=u_{-\mathbf{k}}=0$ and $v_{\mathbf{k}}=v_{-\mathbf{k}}=1$. Since

$$
\left(\tilde{c}_{\mathbf{k} \uparrow}^{\dagger} \tilde{c}_{-\mathbf{k} \downarrow}^{\dagger}\right)\left(\tilde{c}_{-\mathbf{k} \uparrow}^{\dagger} \tilde{c}_{\mathbf{k} \downarrow}^{\dagger}\right)=\left(c_{\mathbf{k} \uparrow}^{\dagger} c_{-\mathbf{k} \downarrow}^{\dagger}\right)\left(c_{-\mathbf{k} \uparrow}^{\dagger} c_{\mathbf{k} \downarrow}^{\dagger}\right)
$$

deep in the Fermi sea the new wavefunction is the same as the BCS wavefunction. This also means that in the normal state $\left(u_{\mathbf{k}} v_{\mathbf{k}}=0\right.$ for all $\left.\mathbf{k}\right)$ the wavefunctions Eq. (4) and Eq. (26) are identical. However, in the region where $u_{\mathbf{k}} v_{\mathbf{k}} \neq 0$ there is a difference. We have for $(\mathbf{k} \uparrow$ $,-\mathbf{k} \downarrow)$ and $(-\mathbf{k} \uparrow, \mathbf{k} \downarrow)$ pairs in the BCS wavefunction

$$
\begin{aligned}
\mid \Psi>_{B C S}^{\mathbf{k},-\mathbf{k}} & =\left(u_{\mathbf{k}}+v_{\mathbf{k}} c_{\mathbf{k} \uparrow}^{\dagger} c_{-\mathbf{k} \downarrow}^{\dagger}\right)\left(u_{-\mathbf{k}}+v_{-\mathbf{k}} c_{-\mathbf{k} \uparrow}^{\dagger} c_{\mathbf{k} \downarrow}^{\dagger}\right) \\
& =u_{\mathbf{k}}^{2}+v_{\mathbf{k}}^{2}\left(\tilde{c}_{\mathbf{k} \uparrow}^{\dagger} \tilde{c}_{-\mathbf{k} \downarrow}^{\dagger}\right)\left(\tilde{c}_{-\mathbf{k} \uparrow}^{\dagger} \tilde{c}_{\mathbf{k} \downarrow}^{\dagger}\right) \\
& -i u_{\mathbf{k}} v_{\mathbf{k}}\left(\left(\tilde{c}_{\mathbf{k} \uparrow}^{\dagger} \tilde{c}_{\mathbf{k} \downarrow}^{\dagger}\right)+\left(\tilde{c}_{-\mathbf{k} \uparrow}^{\dagger} \tilde{c}_{-\mathbf{k} \downarrow}^{\dagger}\right)\right)
\end{aligned}
$$

(assuming as usual $u_{\mathbf{k}}=u_{-\mathbf{k}}, v_{\mathbf{k}}=v_{-\mathbf{k}}$ ). Instead, in the new wavefunction Eq. (26)

$$
\begin{aligned}
\mid \Psi>_{\text {new }}^{\mathbf{k},-\mathbf{k}} & =\left(u_{\mathbf{k}}+v_{\mathbf{k}} \tilde{c}_{\mathbf{k} \uparrow}^{\dagger} \tilde{c}_{-\mathbf{k} \downarrow}^{\dagger}\right)\left(u_{-\mathbf{k}}+v_{-\mathbf{k}} \tilde{c}_{-\mathbf{k} \uparrow}^{\dagger} \tilde{c}_{\mathbf{k} \downarrow}^{\dagger}\right) \\
& =u_{\mathbf{k}}^{2}+v_{\mathbf{k}}^{2}\left(\tilde{c}_{\mathbf{k} \uparrow}^{\dagger} \tilde{c}_{-\mathbf{k} \downarrow}^{\dagger}\right)\left(\tilde{c}_{-\mathbf{k} \uparrow}^{\dagger} \tilde{c}_{\mathbf{k} \downarrow}^{\dagger}\right) \\
& +u_{\mathbf{k}} v_{\mathbf{k}}\left(\left(\tilde{c}_{\mathbf{k} \uparrow}^{\dagger} \tilde{c}_{-\mathbf{k} \downarrow}^{\dagger}\right)+\left(\tilde{c}_{-\mathbf{k} \uparrow}^{\dagger} \tilde{c}_{\mathbf{k} \downarrow}^{\dagger}\right)\right)
\end{aligned}
$$

Eqs. (28) and (29) differ in the last line. The BCS wavefunction has an amplitude for having double occupancy of the state $\tilde{\varphi}_{\mathbf{k}}$ with the state $\tilde{\varphi}_{-\mathbf{k}}$ being empty, and vice versa, and no amplitude for the states $\tilde{\varphi}_{\mathbf{k}}$ and $\tilde{\varphi}_{-\mathbf{k}}$ being both singly occupied. Instead, the new state has amplitude for the states $\tilde{\varphi}_{\mathbf{k}}$ and $\tilde{\varphi}_{-\mathbf{k}}$ being both singlyoccupied and no amplitude for the state $\tilde{\varphi}_{\mathbf{k}}$ being doubly occupied with the state $\tilde{\varphi}_{-\mathbf{k}}$ empty, nor vice versa. Single occupancy of both $\tilde{\varphi}_{\mathbf{k}}$ and $\tilde{\varphi}_{-\mathbf{k}}$ minimizes the direct Coulomb repulsion between the electrons while double occupancy of either state maximizes the direct Coulomb repulsion. Consequently, we conclude that the new wavefunction Eq. (26) will be strongly favored energetically over the BCS wavefunction Eq. (4) as far as the Coulomb interaction between electrons is concerned.

In summary, in the normal state where states $\mathbf{k}$ and $-\mathbf{k}$ are both either occupied or empty, the new basis is completely equivalent to the conventional one. However in a state of the BCS form that allows for partial occupation of pair states the new basis appears to be favorable.

Furthermore, Eqs. (29), (16b) and (14b) indicate that the advantage of the new basis over the conventional one will be greatest if $u_{\mathbf{k}} v_{\mathbf{k}} \neq 0$ in a region where $k$ is small. Instead, for $\mathbf{k}$ near the edge of the Brillouin zone there is no advantage to the new basis according to Eq. (19). This suggests that in a superconducting state described by the wavefunction Eq. (26) the region where $u_{\mathbf{k}} v_{\mathbf{k}} \neq 0$ should occur near $k=0$, and the states with $\mathbf{k}$ near the Brillouin zone edge should be full $\left(v_{\mathbf{k}}=1, u_{\mathbf{k}}=0\right)$ (to conserve the number of particles). This is precisely the scenario predicted in Ref.[6].

\section{4-ELECTRON STATE}

We consider in the following the plane wave case only. Let us consider further the two candidate wavefunctions Eqs. (4) and (26) for the case of only two pairs, in states $(\mathbf{k} \uparrow,-\mathbf{k} \downarrow)$ and $(-\mathbf{k} \uparrow, \mathbf{k} \downarrow)$. The Coulomb energy in the $\mathrm{BCS}$ case is

$$
<V_{c}>_{B C S}=\frac{4 V(0)}{\Omega}\left|v_{\mathbf{k}}\right|^{4}+\frac{2(V(0)+V(2 k))}{\Omega}\left|u_{\mathbf{k}}\right|^{2}\left|v_{\mathbf{k}}\right|^{2}
$$

and with the new wavefunction Eq. (26) it is

$$
<V_{c}>_{\text {new }}=\frac{4 V(0)}{\Omega}\left|v_{\mathbf{k}}\right|^{4}+\frac{2 V(0)}{\Omega}\left|u_{\mathbf{k}}\right|^{2}\left|v_{\mathbf{k}}\right|^{2}
$$

Since the Coulomb repulsion $V(2 k)$ is positive for all $k$, the new state has clearly lower Coulomb energy than the BCS state.

However in adding the second electron pair we have lost the lowering of energy proportional to $V(2 k)$ that occurred for the single pair (Eq. (16b)) due to interference effects. How can it be restored? In deriving Eq. (30), we assumed $u_{\mathbf{k}}=u_{-\mathbf{k}}, v_{\mathbf{k}}=v_{-\mathbf{k}}$. Not making that 
assumption we obtain instead

$$
\begin{aligned}
& <V_{C}>_{B C S}=\frac{V(0)}{\Omega}\left(\left|v_{\mathbf{k}}\right|^{2}+\left|v_{-\mathbf{k}}\right|^{2}\right)^{2} \\
& +\frac{V(0)}{\Omega}\left(\left|u_{\mathbf{k}}\right|^{2}\left|v_{\mathbf{k}}\right|^{2}+\left|u_{-\mathbf{k}}\right|^{2}\left|v_{-\mathbf{k}}\right|^{2}\right) \\
& +\frac{V(2 k)}{\Omega}\left(u_{\mathbf{k}}^{*} v_{\mathbf{k}} u_{-\mathbf{k}} v_{-\mathbf{k}}^{*}+u_{-\mathbf{k}}^{*} v_{-\mathbf{k}} u_{\mathbf{k}} v_{\mathbf{k}}^{*}\right)
\end{aligned}
$$

and with the new wavefunction Eq. (26)

$$
\begin{aligned}
& <V_{c}>_{n e w}=\frac{V(0)}{\Omega}\left(\left|v_{\mathbf{k}}\right|^{2}+\left|v_{-\mathbf{k}}\right|^{2}\right)^{2} \\
& +\frac{V(0)}{\Omega}\left(\left|u_{\mathbf{k}}\right|^{2}\left|v_{\mathbf{k}}\right|^{2}+\left|u_{-\mathbf{k}}\right|^{2}\left|v_{-\mathbf{k}}\right|^{2}\right) \\
& -\frac{V(2 k)}{2 \Omega}\left|u_{\mathbf{k}} v_{-\mathbf{k}}-u_{-\mathbf{k}} v_{\mathbf{k}}\right|^{2}
\end{aligned}
$$

The last term in Eq. (31b) lowers the energy if

$$
\frac{v_{\mathbf{k}}}{u_{\mathbf{k}}} \neq \frac{v_{-\mathbf{k}}}{u_{-\mathbf{k}}}
$$

that is, if the occupation of $(\tilde{\mathbf{k}} \uparrow,-\tilde{\mathbf{k}} \downarrow)$ is different from that of $(-\tilde{\mathbf{k}} \uparrow, \tilde{\mathbf{k}} \downarrow)$. Thus, it favors a spontaneous breaking of parity and the existence of a spin current [9].

In earlier work we have presented several arguments in favor of the suggestion that superconductors possess a spontaneous spin current in their ground state [5, 79]. The direction of the spin current is determined by the spin-orbit interaction in the presence of an outwardpointing electric field predicted by the theory of hole superconductivity [1] together with the sample geometry. Namely, near a surface with outward normal $\hat{\mathbf{n}}$ the member of the Cooper pair of spin $\sigma$ has larger velocity in direction $\hat{\mathbf{n}} \times \vec{\sigma}[9]$. Also, C.R. Hu has argued quite generally that the structure of the BCS state is compatible with the existence of a spin current [12].

\section{COULOMB INTERACTION IN THE PAIR WAVEFUNCTIONS}

The average of the Coulomb interaction in the BCS wavefunction Eq. (4) is given by

$$
<V_{c}>_{B C S}=\frac{1}{\Omega} \sum_{\mathbf{k k}^{\prime}} u_{\mathbf{k}}^{*} v_{\mathbf{k}} u_{\mathbf{k}^{\prime}} v_{\mathbf{k}^{\prime}}^{*} V\left(\mathbf{k}-\mathbf{k}^{\prime}\right)+\frac{V(0)}{\Omega}\left(\sum_{\mathbf{k}}\left|v_{k}\right|^{2}\right)^{2}
$$

and in the new wavefunction Eq. (26) by

$$
\begin{aligned}
<V_{c}>_{n e w} & =\frac{1}{2 \Omega} \sum_{\mathbf{k k}^{\prime}} u_{\mathbf{k}}^{*} v_{\mathbf{k}} u_{\mathbf{k}^{\prime}} v_{\mathbf{k}^{\prime}}^{*}\left[V\left(\mathbf{k}^{\prime}-\mathbf{k}\right)-V\left(\mathbf{k}^{\prime}+\mathbf{k}\right)\right] \\
& +\frac{V(0)}{\Omega}\left(\sum_{\mathbf{k}}\left|v_{k}\right|^{2}\right)^{2} \\
& \left.+\frac{V(0)}{2 \Omega} \sum_{\mathbf{k}}\left(u_{\mathbf{k}}^{*} v_{\mathbf{k}} u_{-\mathbf{k}} v_{-\mathbf{k}}^{*}+\left|u_{\mathbf{k}}\right|^{2}\left|v_{\mathbf{k}}\right|^{2}\right)\right) \\
& +\frac{1}{2 \Omega} \sum_{\mathbf{k}} V(2 k)\left|v_{\mathbf{k}}\right|^{2}\left[\left|v_{-\mathbf{k}}\right|^{2}-\left|v_{\mathbf{k}}\right|^{2}\right)
\end{aligned}
$$

For a clearer comparison we separate self-energy terms in the double sums and obtain for the BCS case

$$
\begin{aligned}
& <V_{c}>_{B C S}=\frac{1}{\Omega} \sum_{\mathbf{k} \neq \mathbf{k}^{\prime}} u_{\mathbf{k}}^{*} v_{\mathbf{k}} u_{\mathbf{k}^{\prime}} v_{\mathbf{k}^{\prime}}^{*} V\left(\mathbf{k}-\mathbf{k}^{\prime}\right) \\
& +\frac{V(0)}{\Omega}\left(\sum_{\mathbf{k}}\left|v_{k}\right|^{2}\right)^{2}+\frac{V(0)}{\Omega} \sum_{k}\left|u_{\mathbf{k}}\right|^{2}\left|v_{\mathbf{k}}\right|^{2}
\end{aligned}
$$

and for the new wavefunction

$$
\begin{aligned}
& <V_{c}>_{\text {new }}= \\
& \quad \frac{1}{2 \Omega} \sum_{\substack{\mathbf{k} \neq \mathbf{k}^{\prime} \\
\mathbf{k} \neq-\mathbf{k}^{\prime}}} u_{\mathbf{k}}^{*} v_{\mathbf{k}} u_{\mathbf{k}^{\prime}} v_{\mathbf{k}^{\prime}}^{*}\left[V\left(\mathbf{k}^{\prime}-\mathbf{k}\right)-V\left(\mathbf{k}^{\prime}+\mathbf{k}\right)\right] \\
& +\frac{V(0)}{\Omega}\left(\sum_{\mathbf{k}}\left|v_{k}\right|^{2}\right)^{2}+\frac{V(0)}{\Omega} \sum_{k}\left|u_{\mathbf{k}}\right|^{2}\left|v_{\mathbf{k}}\right|^{2} \\
& -\frac{1}{4 \Omega} \sum_{\mathbf{k}} V(2 k)\left|u_{\mathbf{k}} v_{-\mathbf{k}}-u_{-\mathbf{k}} v_{\mathbf{k}}\right|^{2}
\end{aligned}
$$

The BCS expression Eq. (35) has the same form whether or not parity is broken. Instead, in the expression Eq. (36) both the first and last term vanish identically if parity is unbroken, and the average of the Coulomb interaction (as well as of any other interaction) gives a self-energy which is identical to that of the BCS case (2nd lines in Eqs. (35) and (36)).

Thus we conclude that there is a stark difference between the BCS wavefunction and the new wavefunction. The BCS wavefunction will give rise to superconductivity if the first term in Eq. (35) is negative on average [3]. This will be the case if the interaction $V\left(\mathbf{k}-\mathbf{k}^{\prime}\right)$ in addition to the repulsive Coulomb interaction contains negative (attractive) terms arising e.g. from the electronphonon interaction, and if the attractive terms are sufficiently large that they can overwhelm the repulsive Coulomb terms. Of course the frequency dependence of the electron-phonon interaction provides additional help by reducing the effective strength of the Coulomb interaction [13].

Instead, with the new wavefunction an attractive interaction (whether instantaneous or retarded) will not help if parity is unbroken, since it will cancel identically in the first term in Eq. (36). The direct Coulomb repulsion is however also eliminated in the first term in Eq. (36) in contrast to the first term in Eq. (35) if parity is unbroken. Furthermore, if parity is broken, the last term in Eq. (36) provides a lowering of energy in the superconducting state for a repulsive interaction, and particularly if the region where $u_{\mathbf{k}} v_{\mathbf{k}} \neq 0$ occurs near $k=0$.

\section{DISCUSSION}

It is natural to try to describe the superconducting state using electronic states that do not optimize the electron-ion interaction and many such attempts have 
been tried in the past. For example, Froehlich [14] proposed a state where a shell of occupied states at the top of the normal Fermi distribution is moved outward, leaving behind an empty shell. This has a certain resemblance to the scenario proposed in Ref. [6]. Even in conventional BCS theory, the normal state Fermi surface is 'blurred' and Bloch states that are empty in the normal state (i.e. unfavorable for the electron-ion interaction) become partially occupied in the BCS state. However we are not aware of earlier attempts to describe the superconducting state using single particle states that violate Bloch's theorem as we propose in this paper, except for the early work of Kronig [15] and Heisenberg [16] that proposed theories of superconductivity based on an electronic 'lattice' that had no relation to the underlying ionic lattice. In addition, Ref. [17] used sine functions to take into account the effect of sufaces and impurities.

As discussed here, the basis introduced in this paper is consistent with the proposal of ref. [6] that in the superconducting state the states near the top of the band / edge of the Brillouin zone become full and those near the bottom of the band / center of the zone, that resemble free-electron-like states, become empty or partially occupied (Fig. 6 of ref. [6]). This is because the new basis gives rise to energy lowering compared with the conventional basis for small $\mathbf{k}$ (near the bottom of the band), while it has no advantage over the conventional basis for $\mathbf{k}$ near the edge of the Brillouin zone (states near the top of the band). This in turn is consistent with the observation that properties of the superconducting state are 'universal', because they arise from long wavelength states near the bottom of the band that are insensitive to details of the electron-ion potential. In order for a system to be able to push the electronic occupation to the top of the band (Fig. 6 of ref. [6]) without undue energy cost requires the band to be almost full in the first place (hole conduction in the normal state), and is favored by a low curvature of the band near the top (large effective mass), a narrow band and a small ionic charge $Z$, which are all requirements that we discussed earlier in connection with the theory of hole superconductivity but based on different arguments.

We also found here that with the new basis the energy is lowered if parity is broken and a spin current exists, consistent with our earlier predictions [5, 9]. In a small cluster, a rigid electronic state where parity is broken should lead to an electric dipole moment as observed in the remarkable experiments of de Heer and coworkers [18].

The new basis proposed here may be of interest also for other unconventional theories of superconductivity besides the theory of hole superconductivity.
[1] F. Bloch, Z. Physik 52, 555-600 (1929).

[2] W. Kohn and L. J. Sham, Phys. Rev. 140, A1133 - A1138 (1965).

[3] J. Bardeen, L. N. Cooper, and J. R. Schrieffer, Phys. Rev. 108, 1175(1957).

[4] J.E. Hirsch, Phys.Rev. B71, 104522 (2005).

[5] J.E. Hirsch, Europhys. Lett. 81, 67003 (2008); J. Phys. Cond. Matt. 20, 235233 (2008).

[6] J.E. Hirsch, Int. J. Mod. Phys. 23, 3035 (2009).

[7] J.E. Hirsch, Phys.Lett.A 309, 457 (2003).

[8] J.E. Hirsch, Phys.Lett.A 315, 474 (2003).

[9] J.E. Hirsch, Phys.Rev. B71, 184521 (2005).

[10] J.E. Hirsch and F. Marsiglio, Phys.Rev. B39, 11515 (1989).
[11] J.E. Hirsch, Phys.Rev. B68, 184502 (2003).

[12] C.R. Hu, arXiv: 0711.3020 (2007).

[13] P. Morel and P.W. Anderson, Phys. Rev. 125, 1263 (1962).

[14] H. Froelich, Phys. Rev. 79, 845 (1950).

[15] R. de L. Kronig, Z. Phys. 78, 744 (1932).

[16] W. Heisenberg, Zeitschrift fur Naturforschung 2a, 185 (1947).

[17] K. Tanaka and F. Marsiglio, Phys.Rev. B 62, 5345 (2000).

[18] S. Yin, S. Xu, A. Liang, J. Bowlan, R. Moro and W.A. de Heer, Jour. of Superconductivity and Novel Magnetism 21, 265 (2008) and references therein. 